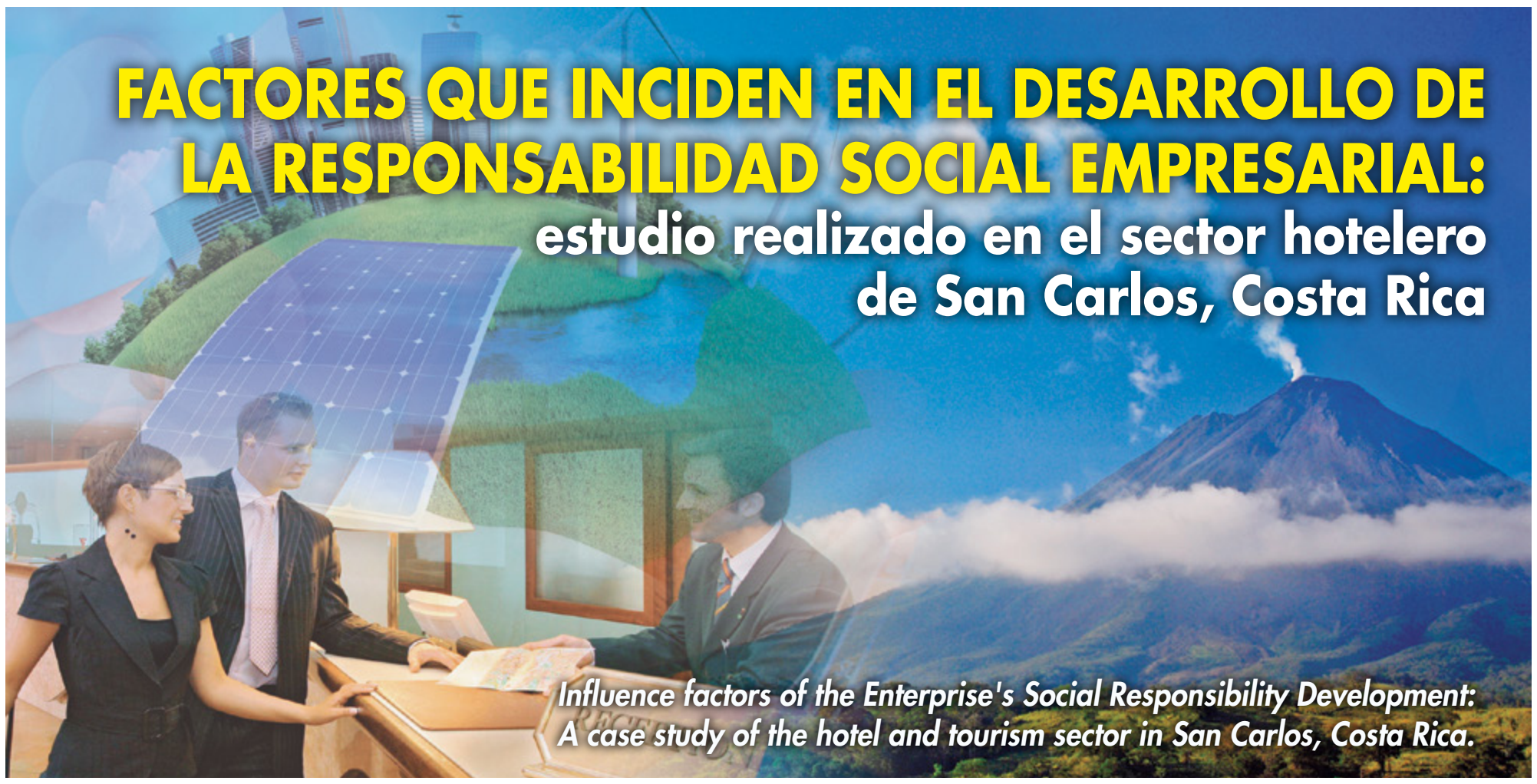

Tec Empresarial, Nov 2015 - Mar 2016, Vol 9 Núm 3 / p. 7-18.

\section{José Martínez-Villavicencio}

jomartinez@ tec.ac.cr

$$
\begin{aligned}
& \text { Doctor en Dirección de Empresas por la Universidad } \\
& \text { de Valencia, España. Profesor catedrático de la Es- } \\
& \text { cuela de Administración de Empresas del Tecnológico } \\
& \text { de Costa Rica. }
\end{aligned}
$$

\section{Ronald Brenes-Sánchez}

rbreness@ ec.ac.cr

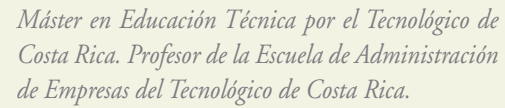

Máster en Educación Técnica por el Tecnológico de Costa Rica. Profesor de la Escuela de Administración de Empresas del Tecnológico de Costa Rica.

\section{Ximena Araneda-Fornachiari} xaraneda@ tec.ac.cr

\section{Máster en Planificación Económica por la Univer- sidad Amistad de los Pueblos, Moscú, Federación Rusa. Profesora de la Escuela de Administración de Empresas del Tecnológico de Costa Rica.}

\section{William Jaubert-Solano} wjaubert@tec.ac.cr

Máster en Dirección de Empresas por la Universidad Latina de Costa Rica. Profesor de la Escuela de Administración de Empresas del Tecnológico de Costa Rica.

- Recepción del artículo: 23 junio, 2015

- Aprobación del artículo: 22 septiembre, 2015

\section{ABSTRACT}

In the last years, small, medium and big companies from Costa Rica have intensified their interest in Corporate Social Responsibility (CSR). Given the sustainability and survival growing urgency in modern society, commercial activity assumes responsible and integral actions on the country development. The aim of this article is to identify the driving factors of CSR, which are, specifically, consumers, suppliers, community, environment, competitiveness and financing, to formulate management models and measurement indicators that could be used to influence and ease the responsible task of a company. For this purpose, we perform a case study within 16 hotel enterprises in La Fortuna de San Carlos, applying a qualitative methodology by semi-structured interview means. Our results indicate that the main driving factor of CSR is the consumers; in second place, the environment, while we found that suppliers and community don't have relevant effect as a driving factor of CSR. This information allows us to support the creation of social policies and sustainable regional tourism.

\title{
RESUMEN
}

En los últimos años, las pequeñas, medianas y grandes empresas de Costa Rica han intensificado su interés en la Responsabilidad Social Empresarial (RSE), debido a que cada vez hay mayor urgencia para la sostenibilidad y sobrevivencia de la sociedad, así como que la actividad comercial asuma acciones de manera responsable e integral para el desarrollo del país. Este estudio tiene por objetivo identificar los factores impulsores de la RSE, identificados, específicamente, como "consumidores", "proveedores", "comunidad", "medio ambiente", "competitividad" y "financiamiento", para formular modelos de gestión e indicadores de medición que puedan ejercer influencia y facilitar que una empresa sea responsable. Para esto se realizó un estudio de casos con dieciséis empresas hoteleras de la Fortuna de San Carlos y se aplicó una metodología cualitativa mediante una entrevista semiestructurada. Dentro de los resultados más importantes se encontró, como principal impulsor de RSE, a los "consumidores" $y$, en segunda instancia, al "ambiente", mientras que se logró identificar que "proveedores" y "comunidad" no tienen mayor efecto como impulsores de RSE, información que permite apoyar la creación de políticas sociales y sostenibilidad turística a nivel regional.

Palabras clave: Responsabilidad social empresarial, factores impulsores, sostenibilidad turística, pymes. $\gg$ 


\section{$\gg$ INTRODUCCIÓN}

Los factores impulsores de la Responsabilidad Social Empresarial (RSE) pueden actuar de forma diferente dependiendo del sector de la economía y de los países, así como de los obstáculos a los que se enfrentan para poder actuar positivamente. Costa Rica requiere transitar sobre el camino de un desarrollo más sostenible y más inclusivo, es por eso que conocer los factores impulsores que motivan la implementación de la RSE en las empresas puede ayudar en esta dirección.

Se ha decidido focalizar el presente estudio al sector turístico, concretamente a la actividad hotelera, al considerar que el turismo se ha convertido en el segundo sector generador de divisas en el país, el cual ha contribuido al Producto Interno Bruto (PIB) durante la última década, con entre un $7 \%$ y un $15 \%$, y genera el $11 \%$ del empleo a nivel nacional. Se ha planteado desarrollar la investigación en San Carlos por ser un cantón en el que destaca la presencia de empresas dedicadas al sector de interés (Pérez, 2001).

Este estudio es relevante debido a que conocer y evaluar los factores que impulsan la incorporación de la RSE en el marco estratégico de la empresa, ofrecerá al país una propuesta para la promoción de estas prácticas en las organizaciones y mejorará la calidad de vida de la sociedad costarricense como un todo. Adicionalmente, esta investigación puede brindar a los tomadores de decisión del sector turístico y particularmente a los actores de la Zona Económica Especial (ZEE) ${ }^{1}$ de San Carlos información que permita impulsar acciones que profundicen la RSE de las empresas hoteleras, y, por ende, un desarrollo sostenible en la zona.

\section{Planteamiento del problema}

Actualmente es fundamental que las organizaciones asuman, responsable e in- tegralmente, las acciones que desarrollan en la producción de bienes y prestación de servicios, así como las externalidades negativas asociadas a éstas. Este proceso no significa sólo cumplir la ley, pues la responsabilidad de las empresas es asumir los resultados de sus decisiones; no es hacer donaciones, no es generar cualquier empleo, es generar empleo digno; no solo pagar impuestos, es también no sobornar. La interrogante fundamental para entender la exigencia de la sociedad actual pareciera ser: ¿Cómo medir el desempeño responsable?

Algunas empresas se han replanteado las relaciones que mantienen con los principales grupos de interés en los entornos en los que desarrollan sus actividades. No obstante, existen serias dificultades para conciliar los comportamientos de responsabilidad social con la lógica individualista de los modelos económicos del mercado.

A nivel internacional, son numerosos los autores que plantean que la RSE no tiene que ser incompatible con la racionalidad económica de mercado y la creación de valor (Nieto, 2008). A nivel nacional, se han dado esfuerzos, por parte de organizaciones y ONG, para formular modelos de gestión e indicadores de medición de la RSE. Sin embargo, en Costa Rica se encuentra poca evidencia sobre los factores que impulsan a las empresas nacionales y a las extranjeras radicadas en el país a desarrollar prácticas responsables, así como la búsqueda de la competitividad a través de la RSE.

\section{Objetivo}

El propósito de este estudio es identificar los factores impulsores de la Responsabilidad Social de las empresas del sector hotelero del cantón de San Carlos y el rol que tienen en su desarrollo elementos que pueden ejercer influencia y facilitar que una empresa sea responsable.

\section{Justificación}

Para identificar los factores impulsores de la RSE, se requiere establecer instrumentos que permitan medir el desempeño responsable de las empresas, así como ayudar y apoyar a las organizaciones, tanto a nivel de unidades productivas como gremiales, a construir esta información que permita identificar los efectos de su accionar, para que puedan tomar decisiones al respecto. El Estado debe establecer estrategias de política pública que incentive las prácticas responsables e inhibir el comportamiento contrario. A la ciudadanía debería facultársele para ejercer su poder de elección informada. Muchos actores de la sociedad deben contribuir a estos procesos y, entre los actores, las universidades públicas -a través de la investigación, la docencia y la vinculación - representan facilitadores fundamentales y acompañantes de las organizaciones que aspiran a responder responsablemente a las demandas que la sociedad exige y crear valor compartido; esto es no solo económico sino social (Porter y Kramer, 2006).

Uno de los instrumentos de política pública del Estado en Costa Rica es el Certificado de Sostenibilidad Turística (CST), perteneciente a un programa del Instituto Costarricense de Turismo (ICT) diseñado para diferenciar empresas turísticas (alojamiento o agencias turísticas) de acuerdo al grado en que sus operaciones se acerquen a un modelo de sostenibilidad, en cuanto a manejo de recursos naturales, culturales y sociales. Su principal objetivo es convertir el concepto de sostenibilidad en algo real, práctico y necesario dentro de la concepción de lo que es la competitividad turística (Ministerio de Economía, Industria y Comercio, Ministerio de Ambiente y Energía, 2010).

El CST ha sido reconocido por la Organización Mundial de Turismo por ser pionero en modificar la forma de hacer 


\section{En los últimos años, las pequeñas, medianas y grandes empresas de Costa Rica han intensificado su interés en la Responsabilidad Social Empresarial (RSE)}

turismo. Dentro de los principales fines de esta certificación se encuentran: ser un instrumento a través del cual Costa Rica se pueda diferenciar en el mundo como destino turístico sostenible, brindar información contable sobre las empresas certificadas y establecer la sostenibilidad como un elemento de competitividad de las empresas. Al mismo tiempo, las empresas que posean la certificación contarán con beneficios, tales como acceso a información sobre tecnologías amigables con el ambiente y toda aquella necesaria para aplicar el estándar del CST según sea su operación; apoyo con material promocional del programa; impulso diferenciado en actividades y ferias turísticas a nivel nacional e internacional; exoneración o descuento en la cuota de participación en ferias internacionales, dependiendo del nivel obtenido de conformidad con el Reglamento de Eventos Internacionales aprobado por el ICT; participación en eventos, seminarios y talleres organizados por la Comisión Nacional de Acreditación; participación diferenciada en el sitio de internet de la Certificación de Sostenibilidad Turística; y prioridad en los programas de certificación (Ministerio de Economía, Industria y Comercio, y Ministerio de Ambiente y Energía, 2010).

Por otra parte, algunas empresas se han replanteado las relaciones que mantienen con los principales grupos sociales en los entornos en los que desarrollan sus actividades. El concepto erróneo de que la RSE consiste en utilizar sus recursos y realizar actividades que estén diseñadas para aumentar sus utilidades (Friedman, 1962) está cambiando; sin embargo, se mantienen serias dificultades para conciliar los comportamientos de responsabilidad social con la lógica individualista de los modelos económicos de mercado, es por eso relevante identificar cuáles son los verdaderos impulsores que generan prácticas responsables en las organizaciones modernas, lo que permitiría que la sociedad civil logre convertirse en un sector empresarial responsable (Vives y Peinado, 2011).

\section{MARCO TEÓRICO}

Las empresas son organizaciones creadas por sus dueños para obtener ganancias. Para el logro de este fin, se constituyen en organizaciones mercantiles que proveen diferentes tipos de bienes y servicios. En Costa Rica, el marco legal regulatorio de la actividad mercantil está dado por el Código de Comercio. A medida que estas unidades mercantiles fueron aumentando su participación e importancia, tanto individual como grupal, en el manejo de los recursos productivos de la sociedad, se hizo evidente que su accionar ya no solo se podría catalogar de interés privado, sino que pasó a ser de interés público (Vives y Peinado, 2011).

Ante este hecho, han sido variados los esfuerzos por definir el comportamiento que la empresa debe tener en la sociedad. Por ejemplo, algunos plantean que el rol de la empresa en la sociedad se circunscribe únicamente a hacer que sus propietarios aumenten sus ganancias. En esta posición se encuentra el economista Milton Friedman, quien escribió: "hay una y sólo una responsabilidad social de la empresa privada: utilizar sus recursos y realizar actividades que estén diseñadas para aumentar sus utilidades" (Friedman, 1962). Otros, como Michael Porter y Mark Krammer (2006), sostienen que el rol de la empresa es crear valor compartido, es decir, crear valor económico al tiempo que crean valor social. Entre estas dos posiciones se pueden encontrar variadas combinaciones.

Ahora bien, el cuestionamiento sobre el rol que debe jugar la empresa en la sociedad actual ha permitido identificar diferentes actores con los que interactúa la empresa, los que la ISO 26000 denomina "partes interesadas", es decir, un individuo o grupo que tiene interés en cualquier decisión o actividad de la organización (INTE/ISO 26000, 2010). Entre estos grupos se podrían citar a los accionistas, organizaciones ambientales, consumidores, proveedores, trabajadores, comunidades, el Gobierno, entes internacionales y otros afines.

La ISO 26000 señala que una empresa responsable es aquella que asume los impactos que sus decisiones y actividades ocasionan en la sociedad y el medio ambiente, a través de un comportamiento transparente y ético; sin embargo, el comportamiento de la empresa y su relación con sus diferentes partes interesadas depende de la relación de poder que puede ejercer sobre ellas; por ejemplo, la relación con sus trabajadores es muy diferente de la relación que puede tener con las fuentes de financiamiento y proveedores, ya que los trabajadores dependen de que la empresa los contrate, mientras que la empresa, para crecer, depende en gran parte de los entes financieros (INTE/ISO 26000, 2010).

La revisión de literatura sobre este tema en específico ha permitido conocer que existen estudios (principalmente de académicos asociados a organizaciones de la sociedad civil) tendientes a conocer los factores que ejercen presión sobre la empresa para que asuma un comportamiento responsable. $\mathrm{Al}$ respecto, se establecen dos grupos de factores: uno asociado a las partes interesadas, $y$ otro, a los elementos que facilitan que $\gg$ 
$\gg$ las empresas puedan ser responsables (Vives y Peinado, 2011).

En un estudio realizado en empresas multinacionales, se define la influencia de los grupos de interés sobre el comportamiento responsable de la empresa, entre los cuales citan: empleados, consumidores, administraciones públicas y la comunidad local (Nieto, 2008). En el mismo estudio se demuestra que el $48 \%$ de los directivos indicó que los empleados son un factor impulsor de la RSE. Si bien es cierto, como los empleados son un público de interés para la empresa, se podría pensar que no necesariamente ejercen influencia en su comportamiento responsable.

\section{Factores que impulsan la RSE en las empresas}

En cuanto a los principales factores impulsores de la Responsabilidad Social Empresarial, se hallaron seis:

\section{- Los consumidores}

Es un agente económico que posee capacidad adquisitiva para poder satisfacer una serie de necesidades a través de los mecanismos de mercado. El consumidor se hace cada vez más exigente, ya no sólo le basta comprar mirando el precio, también son importantes los valores de la empresa que los fabrica: si es una empresa a la cual se le puede considerar buen vecino o no, si es una empresa que cumple con las disposiciones de ley, entre otras. La posibilidad de las redes sociales ha conferido a los consumidores un poder que va en aumento, el cual las empresas no pueden dejar pasar (Rodríguez, 2005).

Las directrices para las empresas multinacionales de la Organización para la Cooperación y Desarrollo Económicos (OCDE), en relación con la RSE, señalan que los consumidores tiene la obligación de promover, garantizar e incentivar a las empresas y las prácticas comerciales responsables, fiscalizando, evaluando, exponiendo y presionando a las empresas, velando por una mejor información a los consumidores y una mayor conciencia de los problemas, así como fomentando una demanda por bienes producidos de una manera socialmente responsable (Rodriguez, 2005).

Para conocer el impacto que estos pueden tener sobre la empresa, se ha propuesto un modelo que se centra en los efectos percibidos por el consumidor de la RSE en su dimensión económica en relación con su comportamiento post-compra. Los re-

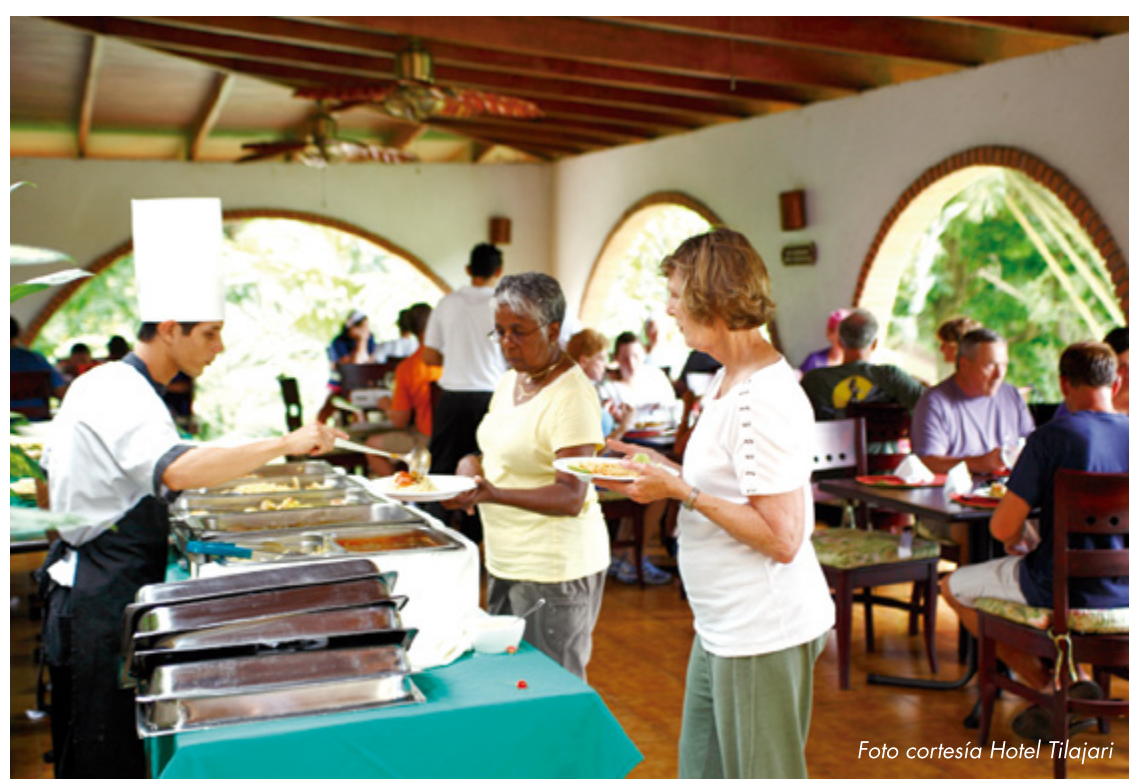

sultados muestran que la percepción de la dimensión económica de la RSE influye, directa y positivamente, sobre la satisfacción del consumidor con la empresa, tanto cognitiva como afectivamente. Asimismo, se confirman los efectos indirectos de la RSE a través de la satisfacción sobre la lealtad hacia la marca (Bigné, Alvarado, Andreu, Aldás y Currás, 2009).

La aplicación de un modelo teórico de relaciones estructurales, contrastado empíricamente a través del análisis de su estructura de covarianzas, en consumidores de la ciudad de Valencia, España, indica que cada una de las dimensiones estudiadas de la responsabilidad social influye significativa y directamente sobre la imagen de la marca, e indirectamente sobre la reputación (Alvarado y Schlesinger, 2008).

\section{- Proveedores}

Actualmente la integración de la RSE dentro de la cadena de valor de las empresas, principalmente en lo que se refiere a los proveedores, se ha convertido en todo un nuevo reto para la gestión (CONFECAMARAS, 2008). Hoy las organizaciones deben preocuparse por hacer o compartir actividades con empresas que integren, dentro de sus operaciones, prácticas responsables o acciones relacionadas con RSE, ya que se convierte en un elemento estratégico y de posicionamiento dentro de un mercado competitivo. Aspectos a tomar en consideración incluyen su reputación, su marca y sus buenas relaciones con otro tipo de organizaciones, no solo dentro del ámbito de su región sino a nivel internacional (Olcese, Rodríguez y Alfaro, 2008).

La visión tradicional de la relación con los proveedores se basa, en primera instancia, en el poder que ejercen sobre las organizaciones. El modelo de las cinco fuerzas planteado por Michael Porter (1980) considera:

- La importancia que puede tener la empresa para el proveedor: entre más importante sea, mayor será su poder. 
- El nivel de diferenciación: entre menos diferenciación tenga el proveedor menor será su poder.

- Menor dificultad en costo del cambio: entre menos cueste cambiar de proveedor, menor será el poder de este.

- Posibilidad de integrar al proveedor: entre mayor probabilidad exista de integrarlo al quehacer de la empresa menor será su poder.

Por otro lado y en segunda instancia, según Olcese, Rodríguez y Alfaro (2008), se puede demostrar la extensión de la RSE a la cadena de suministros, y esto se ha convertido en una característica fundamental de una empresa responsable y sostenible. El nuevo enfoque de la empresa ya no se basa solo en promover a lo interno actividades responsables; sino más bien, es necesario conseguir que el resto de socios que forman parte de la cadena de valor aporten lo que les corresponde.

\section{- Comunidad}

Como lo señalan Vives y Peinado (2011, p. 85), "si la sociedad civil fuera lo suficientemente fuerte y pudiera utilizar los mecanismos adecuados sería uno de los impulsores más poderosos para conseguir un sector empresarial responsable".

En este sentido hay avances muy significativos, como es el ejemplo del Encuentro Nacional sobre Responsabilidad Social Empresarial y Ciudadanía, realizado en Santiago de Chile por un grupo de organizaciones no gubernamentales, donde se concluyó que (Wilson \& Caro, 2007, p. 51):

Ante al reconocimiento de la falta de una cultura de responsabilidad social extendida en las empresas y del relevante papel que éstas podrían jugar en la superación de la pobreza y la inequidad, diversas organizaciones sociales hemos venido desarrollando iniciativas y experiencias tendientes a producir un cambio en el comportamiento empresarial orientado a la implementación de prácticas alineadas con la ética de responsabilidad social.

La acción de una misma parte interesada, como es el caso de la sociedad civil, puede diferir de un país a otro. En contraste con la cita anterior, Mónica Heincke advierte, en un estudio realizado en el 2005 sobre los factores impulsores de la RSE en Colombia, que:

En la sociedad civil tampoco se han dado tantos desarrollos en RSE y los ciudadanos en su mayoría no se han percatado de su importante papel para que la RSE sea un motor en la construcción colectiva de su futuro. Las ONG en Colombia hasta ahora empiezan a incorporar en su óptica y gestión el tema de la RSE con un horizonte estratégico amplio; y centros que se especializan en el tema... (Heincke, 2005, p.69).

\section{- Medio Ambiente}

En un escenario económico deficitario en materia de desarrollo sustentable y una sociedad donde el consumo es un factor que influye en el estilo de vida y el comportamiento de los ciudadanos, las empresas son vistas como actores centrales. La empresa, como organización paradigmática a partir de la era industrial, debe asumir su responsabilidad por el mal uso de recursos no renovables y por las consecuencias de su actividad en el ambiente (Gilli, 2008). De esta manera, el autor plantea que el tema ambiental es un impulsor básico dado que se afecta la base misma de la vida.
Por su lado Porter y Kramer (2006), en "Sociedad y Desarrollo", artículo publicado en Harvard Review en el 2006, habla de cuatro justificaciones predominantes para la Responsabilidad Social, entre las que menciona la sustentabilidad, indicando que la sustentabilidad enfatiza la tutoría medioambiental y comunitaria.

Aunque ya en el 2002, la ESADE, en el "Libro Verde", indica como factores importantes para la empresa la disminución del consumo de recursos, o de los desechos y las emisiones contaminantes, así como la reducción de sus gastos energéticos, la eliminación de residuos y disminuir los insumos y los gastos de descontaminación, distintas empresas han determinado que un menor consumo de materias primas puede redundar en un aumento de la rentabilidad y competitividad.

Por otro lado, la estrategia renovada de la UE para 2011-2014 sobre la responsabilidad social de las empresas menciona la necesidad de reportar sobre los aspectos medioambientales, dadas las crecientes obligaciones que se están incorporando en las legislaciones de los diferentes estados (Comisión Europea, 2011).

\section{- Financiamiento}

Los recursos financieros pueden ser impulsores de los comportamientos empresariales responsables, a través de las diferentes metodologías, instrumentos, índices y tendencias que se dan en los mercados financieros en sostenibilidad social y ambiental. A principios de los años 70, surgieron en los Estados Unidos fondos "éticos" o "responsables" que se abstenían de invertir en algunas empresas o industrias cuestionables. Lógicamente querían ganar dinero, pero dentro de ciertas restricciones coherentes con los principios personales o $\gg$

\section{Se encontró, como principal impulsor de RSE, a los consumidores y en segunda instancia, al ambiente}


$\gg$ institucionales de sus fundadores. Lo curioso fue que estos fondos, que al principio eran sólo un fenómeno marginal y anecdótico, crecieron paulatinamente hasta llegar a tener una relevancia innegable (Saavedra, 2011).

Según el reporte del año 2005 del influyente Social Investment Forum, sólo en los Estados Unidos hay inversiones por 2,3 trillones de dólares invertidos con algún criterio ambiental o social (aproximadamente el $10 \%$ de los fondos administrados en ese país). Si agregáramos las cifras correspondientes a los mercados europeos y de Asia-Pacífico, los números absolutos serían aún más importantes. Entre los países europeos, lideran claramente Holanda y el Reino Unido, teniendo también participaciones relevantes Francia e Italia (Zicari, 2007). La crisis del 2008 deja en evidencia que las fuentes de financiamiento ejercen una gran influencia en el comportamiento responsable o irresponsable de la empresa.

\section{- Competitividad}

La búsqueda de la competitividad estimula las prácticas responsables, centrando el análisis del impacto de las prácticas sociales y ambientales responsables en la gestión empresarial. Los índices de competitividad más recientes muestran que, si bien algunos países latinoamericanos están mejorando rápidamente su potencial para el crecimiento a mediano plazo, otros permanecen estáticos o, incluso, presentan señales de deterioro frente a sus competidores. $\mathrm{La}$ realidad de las condiciones persistentes de pobreza y desigualdad sugiere que la distribución irregular del crecimiento económico no llevará, por sí sola, al desarrollo sostenible. Se requiere una forma más responsable de competitividad (AccountAbility, 2006).

Algunas medidas reguladoras crean un entorno más propicio para que las empresas asuman voluntariamente su responsabilidad social (Comisión Europea, 2011).

Existen varios estudios en los que se busca conocer la relación que tiene RSE y

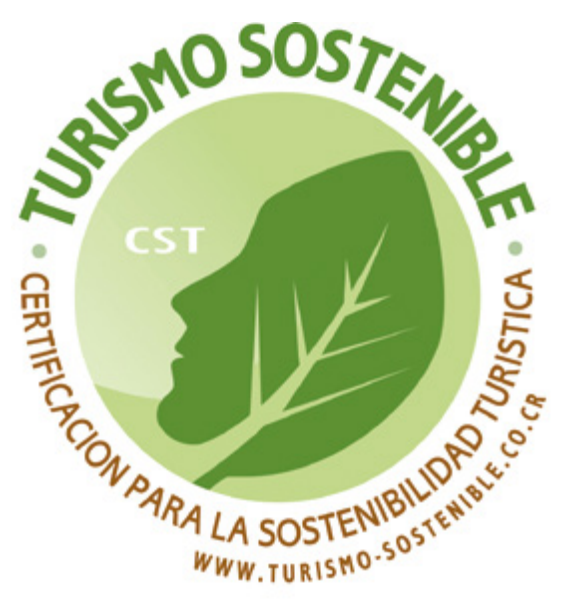

la competitividad. Algunos de estos indican que existe una relación positiva entre el desarrollo de prácticas de RSE y la competitividad de la empresa (Pivato, Misani y Tencati, 2008), incluso se brindan indicadores de que las prácticas de responsabilidad empresarial explican cierta parte de la competitividad de la empresa, tomando en cuenta que la RSE impulsa la innovación y esta, a su vez, impulsa la competitividad (Gallardo y Sánchez, 2013).

Sin embargo, otros estudios indican que no existe una relación lo suficientemente fuerte como para que una explique a la otra (Blowfield y Murray, 2008). Otros señalan que las empresas que son socialmente responsables tienen una desventaja competitiva ya que tienen costos que podrían evitarse (Waddock y Graves, 1997). Existe una creencia de que en un ambiente más competitivo va a haber una baja inversión en RSE, debido a que esto podría afectar la posición económica de la empresa (Fernández y Santaló, 2010).

De acuerdo con una investigación de Cambio Cultural (2004), existe una forma de medir la competitividad en el negocio, basada en factores internos de la empresa y factores externos. Los factores internos se refieren principalmente a sofisticación del proceso de producción, naturaleza de la ventaja competitiva, alcance de la capacitación de los funcionarios, extensión del marketing, disposición a delegar autoridad, capacidad de innovación, gasto en investigación y desarrollo, presencia de cadena de valor, amplitud de los mercados internacionales, grado de orientación al consumidor, control de la distribución internacional, extensión de la política de marcas, confianza en la administración profesional, extensión de la compensación por incentivo, extensión de las ventas regionales y frecuencia del licenciamiento de tecnología extranjera.

También toma en cuenta el ambiente de negocios, principalmente la infraestructura, la infraestructura administrativa, los recursos humanos, la infraestructura tecnológica y los mercados de capital. En este apartado también toma las condiciones de la demanda, las industrias relacionadas y de apoyo; y el contexto para la estrategia y competencia de las empresas.

\section{METODOLOGÍA}

La investigación posee un diseño de tipo exploratorio y descriptivo con un enfoque cualitativo, lo que permite conocer a detalle y profundidad una unidad por medio de una entrevista semiestructurada hecha a los representantes de los hoteles en estudio, siendo dieciséis el número total seleccionado.

\section{Selección de la muestra}

Se identificaron las empresas hoteleras del cantón de San Carlos para realizar el estudio, las cuales debían contar con el Certificado de Sostenibilidad Turística al II Semestre 2014.

Esta certificación fue relevante para la selección de la muestra, pues categoriza y diferencia las empresas turísticas de acuerdo con el grado en que su operación se acerque a un modelo de sostenibilidad ${ }^{2}$ en cuanto al manejo de los recursos naturales, culturales y sociales. 


\section{Identificación y análisis de los} factores impulsores de la RSE

Una vez identificadas las empresas a estudiar, con base en la literatura se seleccionaron seis factores impulsores de la RSE; posteriormente, se diseñó una escala respaldada con la información del marco conceptual, la cual fue aplicada a la muestra seleccionada, con el fin de evaluar estos factores para realizar el análisis correspondiente. Dichos factores se establecieron en áreas temáticas: consumidores, proveedores, comunidad, medio ambiente, competitividad y financiamiento. Los indicadores para la evaluación se construyeron promediando los ítems del cuestionario asociados a cada impulsor y varían en un rango de 0 a 5 , donde 0 es el nivel más bajo y 5 el más alto (Tabla 1).

\begin{tabular}{|c|c|}
\hline $\begin{array}{c}\text { Tabla 1: Cantidad de } \\
\text { ítems por factor }\end{array}$ \\
\hline Factor & $\begin{array}{c}\text { Cantidad } \\
\text { de ítems }\end{array}$ \\
\hline Consumidores & 15 \\
\hline Proveedores & 9 \\
\hline Comunidad & 9 \\
\hline Medio Ambiente & 6 \\
\hline Competitividad & 7 \\
\hline Financiamiento & 5 \\
\hline
\end{tabular}

Fuente: Elaboración Propia.

Subsiguientemente, al utilizar un criterio experto, se clasificaron las empresas según el puntaje obtenido con base en tres categorías (Figura 1), las cuales ilustran el grado de influencia de cada indicador para el desarrollo de prácticas de RSE (según el factor impulsor de RSE).

Se plantea que al tener seis respuestas factibles en la valoración de cada ítem, el término medio de 2,5 constituiría la respuesta mínima aceptable de la influencia del impulsor en las actividades de RSE.

Además, se realizó un análisis correlacional de Pearson sobre los factores para identificar el comportamiento de cada uno de ellos y el grado de asociación de los factores analizados.

\section{RESULTADOS}

Una vez agrupados los datos de la investigación se procede a mostrar los resultados encontrados mediante un análisis descriptivo, un cruce de variables y las correlaciones asociadas a los constructos.

\section{A. Análisis descriptivo de los factores impulsores}

La revisión de literatura llevó a determinar un conjunto de factores que influyen en el desarrollo de actividades de Responsabilidad Social. Se determinaron seis factores que impulsan la RSE: consumidores, proveedores, comunidad, medio ambiente, competitividad y financiamiento

En el estudio se logró determinar los factores que impulsan la RSE en las empresas hoteleras del Cantón de San Carlos que poseen prácticas de Responsabilidad Social según las herramientas estadísticas utilizadas, mostrando como resultado indicadores más fuertes y débiles que incitan la RSE (Tabla 2).

Con los datos obtenidos, se procedió a identificar, para cada uno de los impulsores, la media aritmética y la desviación estándar de los ítems que componía cada elemento (Tabla 3).

Se encontró que los factores consumidores y medio ambiente poseen medias de 3,8 y 3,7 respectivamente, ambas por encima de 3,5, y son los que más impulsan la RSE entre los hoteles de la zona. Los factores proveedores y comunidad son, por el contrario, los que menos inciden en el desarrollo de la RSE, con medias de 1,8 y 2,1 inferiores a 2,5 .
Nivel bajo: puntaje menor o igual a 2,5

Nivel medio: puntaje menor a 3,5 y mayor a 2,5

Nivel alto: puntaje mayor a 3,5

Fira 1: Categorías de las evaluaciones de los indicadores de RSE

Una vez considerados los resultados el grupo de los seis impulsores estudiados, se presenta a continuación los detalles encontrados para cada uno de ellos:

\section{- Consumidores}

Este indicador muestra la mayor calificación de los seis impulsores estudiados al ser su media de 3,8, por lo cual se determina que es el principal impulsor de RSE en los hoteles trabajados en el cantón de San Carlos. Únicamente para una de las dieciséis empresas hoteleras estudiadas no es relevante debido a que su indicador es de 1,7, que se encuentra por debajo del nivel deseable en esta investigación, el cual es 3,5 o más para ser catalogado como un factor de alto impacto (Figura 1). Otro elemento relevante está relacionado con la variabilidad de los puntajes de los indicadores, pues se encontró que en el caso de consumidores hay una desviación estándar de 0,9 (Gráfico 1), lo cual muestra que las empresas se comportan de manera muy similar con relación a este ítem.

Además, en cuanto a efectos positivos es posible enumerar los siguientes: las listas de contactos de clientes se consiguen de forma legítima, lo cual es obligatorio y parte de la legalidad de la empresa; la ley 7600, como política pública, obliga a los hoteles a cumplir una serie de condiciones de infraestructura, al igual que los requerimientos internacionales del servicio. $\gg$ 
Tabla 2: Evaluación cuantitativa de los indicadores impulsores de la RSE analizados en el estudio

\begin{tabular}{|c|c|c|c|c|c|c|}
\hline Empresa & Consumidores & Proveedores & Comunidad & Medio Ambiente & Competitividad & Financiamiento \\
\hline 1 & 2,9 & 0,0 & 1,1 & 4,5 & 1,0 & 0,8 \\
\hline 2 & 3,7 & 2,8 & 2,6 & 3,0 & 2,1 & 2,6 \\
\hline 3 & 3,5 & 3,4 & 0,0 & 4,5 & 2,6 & 2,0 \\
\hline 4 & 2,9 & 3,8 & 0,9 & 5,0 & 2,1 & 2,6 \\
\hline 5 & 4,1 & 1,0 & 4,2 & 5,0 & 2,1 & 4,0 \\
\hline 6 & 4,3 & 1,2 & 1,6 & 3,5 & 2,9 & 4,0 \\
\hline 7 & 4,0 & 1,5 & 2,1 & 3,0 & 1,6 & 2,4 \\
\hline 8 & 4,8 & 2,4 & 4,6 & 4,8 & 3,3 & 2,6 \\
\hline 9 & 3,3 & 0,0 & 0,5 & 0,5 & 1,7 & 1,6 \\
\hline 10 & 4,8 & 3,6 & 4,6 & 4,2 & 2,9 & 4,0 \\
\hline 11 & 4,5 & 2,9 & 3,6 & 2,8 & 2,6 & 2,2 \\
\hline 12 & 3,5 & 0,0 & 1,4 & 4,8 & 1,9 & 1,2 \\
\hline 13 & 4,8 & 1,4 & & 4,2 & 3,6 & 1,6 \\
\hline 14 & 4,7 & 2,6 & 1,1 & 4,3 & 2,1 & 2,0 \\
\hline 15 & 2,6 & 1,3 & 1,4 & 3,8 & 2,4 & 1,4 \\
\hline 16 & 1,7 & 0,5 & 0,3 & 1,0 & 1,0 & 2,0 \\
\hline
\end{tabular}

Fuente: Elaboración Propia.

Tabla 3: Media y desviación según cada indicador de los factores estudiados

\begin{tabular}{|c|c|c|}
\hline Indicadores & Media & Desviación \\
\hline Consumidores & 3,8 & 0,9 \\
\hline Proveedores & 3,7 & 1,4 \\
\hline Comunidad & 2,3 & 1,0 \\
\hline Medio Ambiente & 2,2 & 0,7 \\
\hline Competitividad & 2,0 & 1,5 \\
\hline Financiamiento & 1,8 & 1,2 \\
\hline
\end{tabular}

Fuente: Elaboración Propia.

$\gg$ Dentro de los efectos negativos se obtuvo que no se agregan argumentos de RSE en las campañas de marketing y esto podría deberse a que la zona, por sí misma, es percibida como ambientalmente limpia. Por otro lado, no se consigna la RSE en la estrategia empresarial de los hoteles, lo cual debería ser esencial en sus planes de desarrollo. Tampoco, según el estudio, se podría confirmar que los clientes reciben suficiente información para aclarar dudas respecto a la operación del servicio.

\section{- Medio ambiente}

En el caso del medio ambiente como impulsor de la RSE, se muestra una calificación media de 3,7 que se encuentra por encima de 3,5 como calificación de referencia para impulsores de alto impacto, esto indica que representa un impulsor importante de la RSE en los hoteles del cantón de San Carlos.

En dos de las empresas participantes del estudio se obtuvo calificaciones máximas de 5; sin embargo, debe mencionarse que también se encontró una empresa con una media de 0,5 y otra de 1 . La desviación estándar alcanzó el grado de 1,4, lo que indica que las respuestas son bastante dispersas entre los diferentes hoteles que participaron en la investigación.

Dentro de la composición del impulsor, los ítems de mayor evaluación en promedio están relacionados con la variable de si la empresa ha sido auditada en la prevención de la contaminación y cumplimiento de leyes, así como si cuenta con un plan de emergencias ambientales debidamente revisado y que se ajuste a las exigencias públicas.

Por otro lado, los componentes que obtuvieron las menores calificaciones en promedio fueron: la práctica de las empresas de brindar a sus clientes y consumidores información acerca de los daños ambientales que pueden generarse de sus procesos a lo largo en la cadena de valor, y también elementos asociados a la presión de los hoteles a participar en comités locales o regionales para discutir sobre el medio ambiente.

\section{- Proveedores:}

Una vez obtenidos los datos del impulsor denominado proveedores se encuentra la media más baja de todos los estudiados con 1,8, que, al considerar la 


\section{Los proveedores y comunidad no tienen mayor efecto como impulsores de RSE}

Gráfico 1: Media y desviación según cada indicador de los factores estudiados

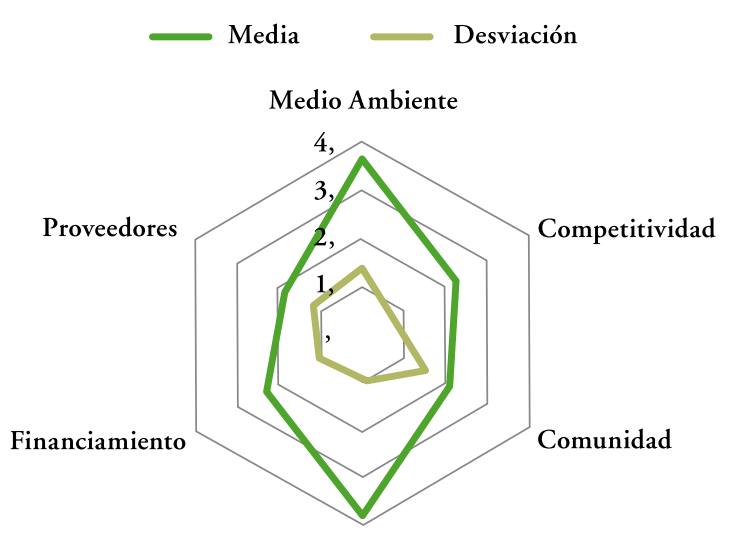

Consumidores dores tienen un código de ética en el que se pide a los hoteles que realicen acciones que los reconozcan.

\section{- Comunidades:}

La valoración de este impulsor se encuentra por debajo del factor de referencia al obtener tan solo 2,1 como media en todos los hoteles estudiados, con lo cual no representa un factor relevante para el desarrollo de actividades de RSE. Dentro de los hoteles investigados, tan solo en dos de los casos se obtuvo una calificación de referencia de 2,5 , se ubica muy por debajo y desde luego muestra que este no representa un impulsor para el desarrollo de las actividades de RSE en los hoteles estudiados.

De las dieciséis empresas, en tres de los casos la media obtenida fue de $0 \mathrm{y}$ tan solo en dos de los sujetos de estudio se obtuvo índices superiores a 3,5, que desde el punto de vista de la calificación de referencia ofrecen un resultado satisfactorio según la dinámica de la investigación.

En el estudio, la desviación estándar obtiene, para este impulsor de la RSE, un valor de 1,2 , lo que indica que los datos de todas las empresas se encuentran muy dispersos.

La mejor calificación en este factor se refiere a la inclusión de las organizaciones comunales y las minorías dentro de su esquema de proveedores. Los factores de menor evaluación se asocian a la práctica de que los proveedores de los hoteles desarrollen visitas de inspección o verificación de prácticas responsables, y si los provee- calificación por encima de 3,5, y las más bajas, 0 y 0,3 en dos de los casos, esto para las dieciséis empresas involucradas.

En la consideración de la variabilidad de los datos se encuentra una desviación estándar de 1,5, lo que indica una alta dispersión de la información con relación a todas las empresas, y representa el dato más elevado con relación a todos los impulsores valorados en el estudio.

El ítem asociado a la comunidad que destacó en la evaluación es el relacionado con la conciencia de que la comunidad debería participar en la identificación de los problemas y conminar a los hoteles a llevar a cabo esta tarea; por otro lado, la solicitud de la comunidad para el desarrollo de programas de RSE por parte del hotel ha sido el ítem con la valoración más baja.

\section{- Financiamiento}

Con respecto a este impulsor de RSE, los datos muestran que la media asciende a 2,3, encontrándose por debajo del dato de referencia en la investigación, que es de 2,5 , con lo cual este no puede considerarse un impulsor en los hoteles del cantón de San Carlos.

De las dieciséis empresas estudiadas, en dos de los casos se encontraron valores de 1,8 y de 1,2, y tan solo en dos de los hoteles se obtuvo un valor de 4 en la calificación de financiamiento como impulsor de la RSE.

Desde la perspectiva de la desviación estándar, el valor encontrado es de 1 , lo que muestra una alta dispersión de la información entre todas las empresas estudiadas.

Como ítem de mayor puntaje en el análisis del financiamiento se encuentra la consideración de la empresa de criterios sociales, éticos o ambientales en la selección de fuentes de financiamientos, mientras que el ítem evaluado con el menor puntaje es si la empresa acepta socios inversionistas que han sido cuestionados ética o moralmente.

\section{- Competitividad}

En el procesamiento de datos se encuentra que la media de las respuestas de las dieciséis empresas es de 2,2, que no alcanza el mínimo de referencia de 2,5 establecido en este trabajo, con lo cual la competitividad no impacta el desarrollo de actividades de RSE de los hoteles del cantón de San Carlos.

La desviación estándar es de 0,7 , lo cual debe interpretarse como una baja dispersión y un mejor agrupamiento de las respuestas encontradas.

En la composición de los ítems que se evaluaron para el factor competitividad, el valor más alto está asociado a la percepción de la empresa de la existencia de una $\gg$ 
$\gg$ relación positiva entre las prácticas de responsabilidad y la competitividad; y el ítem más bajo se asocia a la acción de la empresa para hacer aportes a candidatos o campañas políticas.

\section{B. Análisis correlacional de los factores}

Para establecer el grado de asociación que existe entre los impulsores analizados, se llevó a cabo una correlación de Pearson de los factores.

Los impulsores que se correlacionan linealmente son: consumidores con competitividad, proveedores con competitividad, comunidad con consumidores, competitividad con financiamiento, y medio ambiente con consumidores (Tabla 4). Se evidencia una fuerte dependencia lineal positiva con coeficientes de correlación $r>0,5$ que existe entre estos.

Esta correlación existente indica que, conforme aumenta o disminuye un factor, se incrementa o disminuye los factores correlacionados y viceversa. Este comportamiento se presenta en los factores mencionados con un coeficiente mayor a 0,5 .

Por otro lado, las correlaciones menos acentuadas se dan para los impulsores “ambiente con financiamiento". Es decir, la relación entre estos es muy baja, casi de 0 .
Así como "proveedores con comunidad", donde la relación es moderada.

La relación más alta se da entre competitividad con consumidores, y comunidad con consumidores (Tabla 4).

\section{DISCUSIÓN Y CONCLUSIONES}

De acuerdo con el análisis de la literatura universal sobre los elementos o factores que impulsan a las organizaciones a desarrollar actividades de RSE, se ha encontrado un grupo específico que se puede categorizar como impulsores de RSE: consumidores, proveedores, comunidad, medio ambiente, competitividad y financiamiento.

Para el caso de la variable "consumidores", la literatura muestra componentes concretos que integran aspectos asociados a este factor, entre ellos, las campañas de marketing, libros de quejas y bases de datos de consultas.

Por otro lado, el impulsor denominado "proveedores" integra aspectos asociados, como, por ejemplo, compromisos con requerimientos fiscales y normativas ambientales, competencia desleal y políticas de colaboración o cooperación en las cadenas productivas.

En el caso del impulsor "comunidad", se encontraron componentes como la participación en la identificación y diagnóstico de problemas sociales de la zona, programas de voluntariado donde participan activamente miembros de las empresas y participación en programas de salud y alimentación.

El "medio ambiente" se ha catalogado en la literatura como un elemento fundamental para el desarrollo de actividades de RSE, dentro de las cuales se relacionan prevención de la contaminación, cumplimiento de las leyes ambientales nacionales e internacionales, información de los daños ambientales que pueden resultar de la cadena de valor y planes de emergencias ambientales.

También, la "competitividad" es mencionada en la literatura como un impulsor de la RSE, entre cuyos elementos se puede enumerar: prácticas de RSE en el incremento de la posición en el mercado y productividad de la empresa, ventajas competitivas por costos adicionales y cumplimiento de obligaciones legales y tributarias.

Finalmente, el "financiamiento" genera, según la literatura, algún impacto para el desarrollo de actividades de RSE, por ejemplo, incorporación de socios inversionistas y la selección de fuentes de financiamiento.

Tabla 4: Coeficiente de correlación entre los factores

\begin{tabular}{|c|c|c|c|c|c|c|}
\hline & Consumidores & Proveedores & Comunidad & Medio Ambiente & Competitividad & Financiamiento \\
\hline Consumidores & 1 & & & & & \\
\hline Proveedores & ,439 & 1 & & & & \\
\hline Comunidad & ,709 & ,344 & 1 & & & \\
\hline Medio ambiente &, 510 & ,403 & ,391 & 1 & & \\
\hline Competitividad & ,772 & ,523 & ,598 & ,497 & 1 & \\
\hline Financiamiento & ,418 & ,417 & ,594 & ,172 & ,367 & 1 \\
\hline
\end{tabular}

Fuente: Elaboración Propia. 
Desde el punto de vista de análisis de datos, y después de aplicar una escala para medir el impacto de todos los factores mencionados en la literatura, se agruparon las respuestas y se obtuvieron las medias asociadas a cada uno de los factores, tras lo cual se concluyó que, para los hoteles del cantón de San Carlos, los que impulsan de manera importante a nivel alto, en criterio experto de esta investigación, son "consumidores" y "medio ambiente". Se ha establecido un puntaje superior a 3,5 para considerar a un impulsor como de alto impacto, lo que convierte a estos en impulsores de la RSE en el cantón de San Carlos.

Cuando se analizan los resultados de "financiamiento" y "competitividad" se encuentra que su impacto es de nivel medio, ya que, según el rango establecido para el estudio, es de entre 2,5 y 3,5 , con lo cual también pueden considerarse estos factores como impulsores de la RSE en los hoteles del cantón de San Carlos.

Aun cuando se han considerado dichos resultados en el estudio conceptual, el análisis de la información obtenida en esta investigación arroja que "comunidad" y "proveedores" no representan impulsores de las actividades de RSE en los hoteles del cantón de San Carlos bajo estudio, debido a que los índices de impacto, según las medias encontradas, han sido clasificadas en la categoría de bajo impacto, con rangos inferiores a 2,5. Debe mencionarse que en este caso la desviación estándar es relevante, pues indica una variabilidad alta. Llama la atención que, precisamente, la sociedad en su conjunto es quien recibe las consecuencias negativas de la irresponsabilidad de las empresas.

\section{FUTURAS LÍNEAS DE INVESTIGACIÓN}

Por la naturaleza del tema y la importancia para el desarrollo de las empresas y la economía de los países, se considera relevante el complementar los hallazgos de

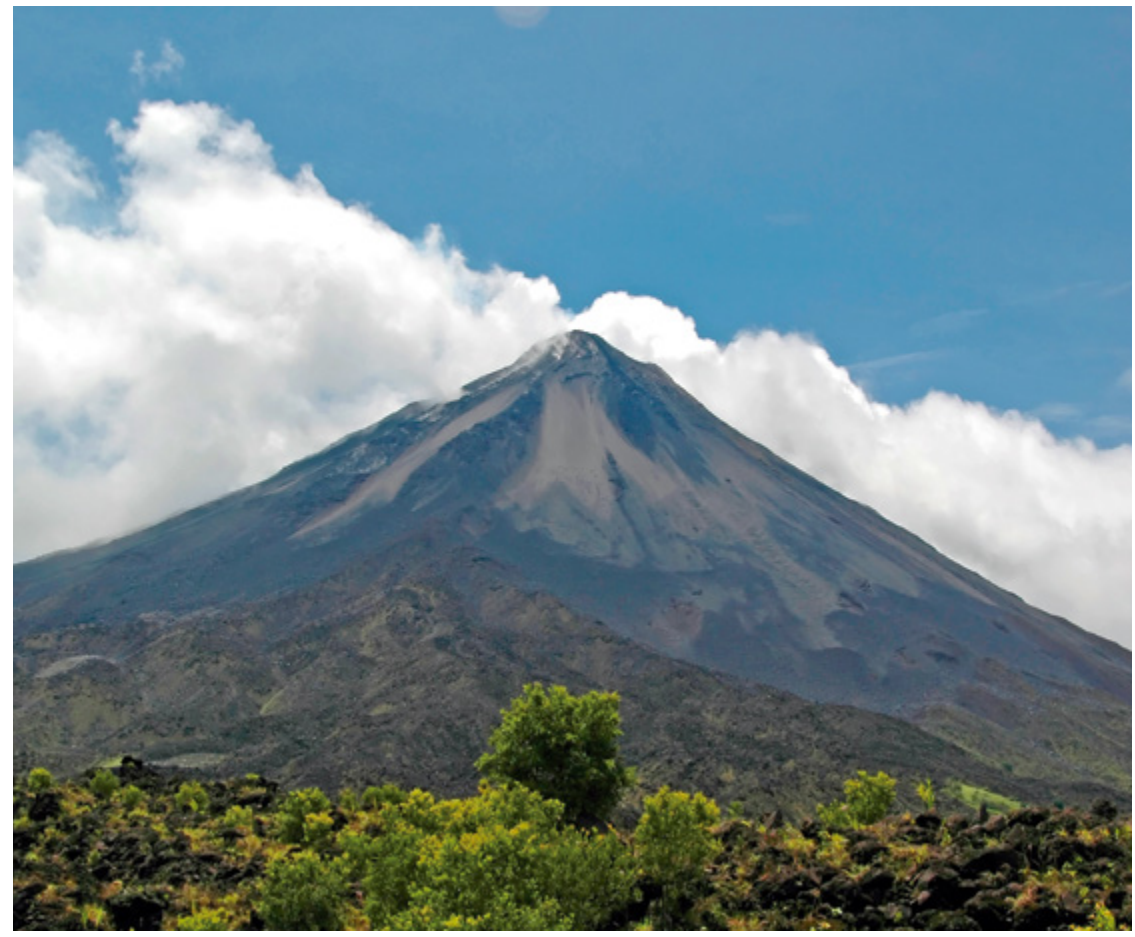

la investigación con otro conjunto de estudios que fortalezcan o que contradigan la información suministrada en este artículo.

- La investigación mostrada se realizó en un segmento particular en el cantón de San Carlos, para la cual se tomó como base la construcción de una escala de valoración determinada por los investigadores, al clasificar, según medias obtenidas, la importancia de los impulsores en bajo, medio y alto. Asimismo, es importante realizar una valoración estadística de esta escala, evaluando otros cantones del país y otras actividades de negocio diferentes a la hotelería.

- En este estudio se aplicó un cuestionario a un grupo de hoteles del cantón de San Carlos, y mediante el análisis de medias se clasificó la importancia de los impulsores, por lo que es factible ampliar este trabajo a diferentes cantones y provincias, y adicionalmente a otras áreas de negocio diferentes a la hotelería, brindando mayor consistencia a los resultados obtenidos y la posibilidad de aplicar un conjunto más diverso de instrumentos estadísticos para el análisis de la información.

- La comunidad y proveedores se han considerado en el resultado de este estudio como factores que no impulsan la RSE en el cantón de San Carlos, por lo que debe realizarse una investigación a profundidad a nivel teórico y práctico que justifique y explique el fenómeno encontrado.

\section{Referencias Bibliográficas}

AccountAbility. (2006). Responsible Competitiveness Index 2006. ( ) AccountAbility \& FDC 2006.http://www.accountability. org/images/content/3/1/319/Competitividad\%20ResponsableIndiceAL2006.pdf

Alvarado, A. y Schlesinger, M. (2008). Dimensionalidad de la responsabilidad social empresarial percibida y sus efectos sobre la imagen y la reputación: una aproximación desde el modelo de Carroll. Estudios Gerenciales, 24 (108). 
\ Bigné, J., Alvarado, A., Andreu, L., Aldás, J., y Currás, R. (2009). Influencia de la percepción de la dimensión económica de la RSE sobre la satisfacción y la lealtad del consumidor. Revista de responsabilidad social de la empresa, (1).

Blowfield, M. \& Murray, A. (2008). Corporate Social Responsibility. A Critical Introduction. Oxford: Oxford University Press.

Cambio Cultural. (2004). Cómo se mide la competitividad. Buenos Aires: Cambio Cultural.

Comisión Europea. (2011). Estrategia renovada de la UE para 2011-2014 sobre la responsabilidad social de las empresas. Disponible en http://es.scribd.com: http:// es.scribd.com/doc/74799840/RSE-NuevaEstrategia-de-La-UE-Para-La-RSE

CONFECAMARAS. (2008). Informe de Presidencia de Gestión 2007-2008.

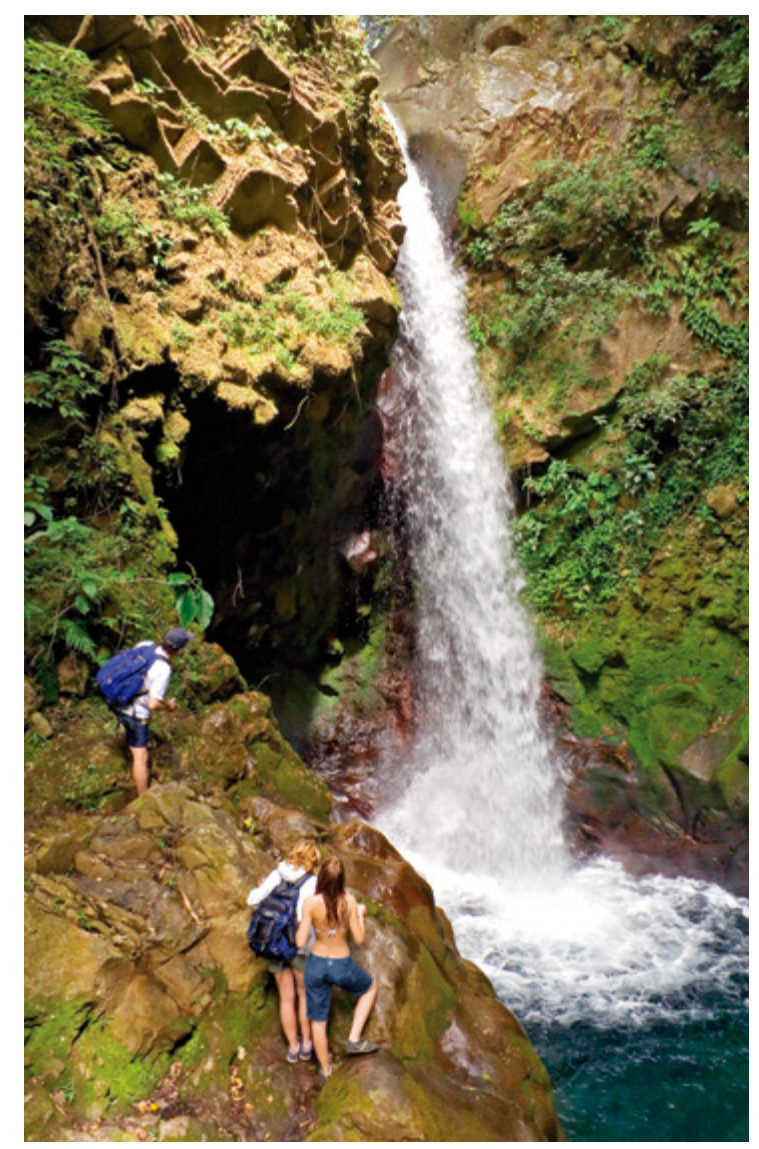

Fernández, D., \& Santaló, J. (2010). When Necessity Becomes a Virtue: The Effect of Product Market Competition on Corporate Social Responsibility. Journal Of Economics \& Management Strategy, 19(2), 453-487.

Friedman, M. (1962). Capitalismo y Libertad. Chicago: Imprenta Universitaria de Chicago.

Gallardo-Vázquez, D., y Sánchez-Hernández, M. (2013). Análisis de la incidencia de la Responsabilidad Social Empresarial en el éxito competitivo de las microempresas y el papel de la innovación. (Spanish) (cover story).Universia Business Review, (38), 1431.

Gilli, J. (2008). Responsabilidad Empresaria y Medio Ambiente. Facultad de Ciencias Económicas - UBA.

Heincke, M. (2005). La responsabilidad social empresarial: una herramienta para el desarrollo local de Colombia. Opera. publicación anual del Observatorio de Políticas Públicas de la Facultad de Finanzas, Gobierno y Relaciones Internacionales de la Universidad Externado de Colombia, 55-74.

INTE/ISO 26000. (2010). Guía de Responsabilidad Social. Costa Rica.

IPES-ESADE. (2002). Libro Verde de la Comisión Europea. Fomentar un marco europeo para la responsabilidad social de las empresas. Barcelona: Diario Oficial de la Comunidades Europeas.

Ministerio de Economía, Industria y Comercio, Ministerio de Ambiente y Energía. (07 de Abril de 2010). Reglamento para el Otorgamiento del Certificado de Sostenibilidad
Turística. Diario Oficial La Gaceta, (131).

Nieto, M. (2008). La difusión de las prácticas de responsabilidad social en las empresas multinacionales. Pecvnia, 33-64.

Olcese, A., Rodríguez, M. y Alfaro, J. (2008). Manual de la Empresa Responsable y Sostenible. Madrid: McGRAW-HILL.

Pérez, J. (2001). Globalización y Comunidades en Centro América. Cartago: FLACSO.

Pivato, S., Misani, N. \& Tencati, A. (2008), The impact of corporate social responsibility on consumer trust: the case of organic food. Business Ethics: A European Review, (17), 3-12.

Porter, M. (1980). Competitive Strategy: Techniques for Analyzing Industries and Competitors. New York: Free Press.

Porter, M. y Kramer, M. (2006). Estrategia y Sociedad. Harvard Business Review, 3-15.

Rodriguez, M. (2005). La responsabilidad social empresaruial y los consumidores. Revista de economóa pública, social y cooperativa, CIRIEC España, 97-109.

Saavedra, M. (2011) La responsabilidad social empresarial y las finanzas. Cuadernos e Administración, 27(46), 39-54.

Vives, A. y Peinado, E. (Eds.) (2011). La responsabilidad social de la empresa en América Latina. Nueva York: Banco Interamericano de Desarrollo.

Waddock, S. \& Graves, S. (1997). The Corporate Social Performance-Financial Performance Link. Strategic Management Journal, 18 (4), 303-319.

Wilson, R. y Caro, P. (2007). Ciudadanía y Responsabilidad Social Empresarial:balance de experiencias y desafios de organizaciones de la sociedad civil. Santiago de Chile: Impresos Socias Ltda.

Zicari, A. (2007). Responsabilidad Social Empresaria, un enfoque financiero. Buenos Aires: Edicon. T 